\title{
Identification And Validation of A Cigarette Smoke- Related Five-Gene Signature As A Prognostic Biomarker In Kidney Renal Clear Cell Carcinoma
}

\section{Yefei Huang}

Xuzhou Medical University

Qinzhi Wang

Xuzhou Medical University

\section{Yu Tang}

Xuzhou Medical University

Zixuan Liu

Xuzhou Medical University

Guixiang Sun

Xuzhou Medical University

Zhaojun Lu

Xuzhou Medical University

Yansu Chen ( 100002014013@xzhmu.edu.cn )

Xuzhou Medical University

\section{Research Article}

Keywords: kidney renal clear cell carcinoma, methylnitrosamino, exposure

Posted Date: July 1st, 2021

DOl: https://doi.org/10.21203/rs.3.rs-593776/v1

License: (9) (i) This work is licensed under a Creative Commons Attribution 4.0 International License.

Read Full License

Version of Record: A version of this preprint was published at Scientific Reports on February 9th, 2022. See the published version at https://doi.org/10.1038/s41598-022-06352-y. 


\section{Abstract}

Cigarette smoking greatly promotes the progression of kidney renal clear cell carcinoma (KIRC), however, the underlying molecular events has not been fully established. In this study, RCC cells were exposed to the tobacco specific nitrosamine 4-(methylnitrosamino)-1-(3-pyridyl)-1-butanone (NNK, nicotine-derived nitrosamine ketone) for 120 days, and then the soft agar colony formation, wound healing and transwell assays were used to explore characteristics of RCC cells. RNA-seq was used to explore differentially expressed genes. We found that NNK promoted RCC cell growth and migration in a dose-dependent manner, and RNA-seq explored 14 differentially expressed genes. In TCGA-KIRC cohort, Lasso regression and multivariate $\mathrm{COX}$ regression models screened and constructed a five-gene signature containing ANKRD1, CYB5A, ECHDC3, MT1E, and AKT1S1. This novel gene signature significantly associated with TNM stage, invasion depth, metastasis, and tumor grade. Moreover, when compared with individual genes, the gene signature contained a higher hazard ratio and therefore had a more powerful value for the prognosis of KIRC. A nomogram was also developed based on clinical features and the gene signature, which showed good application. Finally, AKT1S1, the most crucial component of the gene signature, was significantly induced after NNK exposure and its related AKT-mTOR signaling pathway was dramatically activated. Our findings supported that NNK exposure would promote the KIRC progression, and the novel cigarette smoke-related five-gene signature might serve as a highly efficient biomarker to identify progression of KIRC patients, AKT1S1 might play an important role in cigarette smoke exposure-induced KIRC progression.

\section{Introduction}

Kidney and renal pelvis cancer is among the top ten most common cancers in the world, with 65340 new cases and 14970 deaths in 2018 in United States ${ }^{1}$. Renal cell carcinoma (RCC) accounts for $~ 85 \%$ of all renal malignancies, and kidney renal clear cell carcinoma (KIRC) arising from the proximal convoluted tubule is the most common and malignant histological subtype and responsible for most of deaths among all the subtypes of RCC ${ }^{2}$.

The link between cigarette smoking and RCC has been well-established. Active smoking is associated with histological RCC subtype, especially with KIRC ${ }^{3,4}$. The relative risk of KIRC is not only higher in smokers as compared to non-smokers, but also increased with the cumulative dose and duration of smoking ${ }^{5,6}$. Moreover, cigarette smoking has an ongoing effect on the progression of KIRC. Studies have validated that tobacco exposure is positively associated with aggressive clinical parameters of KIRC and decreases the cancer-specific survival and overall survival of KIRC patients ${ }^{7,8}$. However, the underlying molecular events of cigarette smoking on KIRC progression needed a further study.

High-throughput RNA-seq data have been widely used to elucidate underlying mechanisms and identify prognostic genes for various cancers. In this study, 4-methylnitrosamino-1-3-pyridyl-1-butanone (NNK), the major component of cigarette smoke and the most potent carcinogen among tobacco-specific nitrosamines, was used to stimulate the RCC cells, and then the RNA-seq was performed in the KIRC cell 
line to identify the hub genes closely associated with NNK-induced malignancy. The Gene Ontology (GO) analysis was used to enrich the genes which had the similar function according to the gene expression profiles. Next, the key candidate biomarkers were identified in the KIRC cohort of The Cancer Genome Atlas (TCGA) data. Thereafter, the gene signature was also constructed and its relationship some clinical traits and overall survival of KIRC patients was confirmed. Our work yielded a novel gene signature which was associated with tobacco smoke exposure and can accurately predict its relationship with KIRC progression.

\section{Results}

\section{NNK exposure increased growth and migration abilities of RCC cells.}

The human RCC cell lines 786-0 and KETR-3 were continuously exposed to $0.1 \%$ DMSO and NNK (0.01 and $0.1 \mu \mathrm{M}$ ) for 120 days (40 passages). Then the results of soft agar colony formation assay revealed that the number of cell colonies had a significant dose-dependent increase after NNK exposure when compared with the $0.1 \%$ DMSO control group (Fig. $1 \mathrm{~A}$ and $\mathrm{B}$ ). The wound healing assay was performed and showed that long-term NNK exposure significantly promoted KETR-3 and 786-0 cell migration ability (Fig. 1C-F). The cell transwell assay also was performed and observed that the number of cell migration was significantly increased in a dose-dependent manner after long-term NNK exposure in both KETR-3 and $786-0$ cells when compared with the respective $0.1 \%$ DMSO controls (Fig. $1 \mathrm{G}$ and $\mathrm{H}$ ).

\section{Identification of cigarette smoke exposure-related genes in RCC cells.}

We performed the RNA-seq on $0.1 \%$ DMSO, $0.01 \mu \mathrm{M}, 0.1 \mu \mathrm{M}$ NNK treated $786-0$ cells to explore the potential molecular mechanism in NNK-induced malignancy of RCC cells. The volcano plot identified 389 differentially expressed genes (163 up-regulated and 226 down-regulated genes) in $0.01 \mu \mathrm{M} N N K$ treated group and 418 differentially expressed genes (168 up-regulated and 250 down-regulated genes) in $0.1 \mu \mathrm{M}$ NNK treated group when compared with $0.1 \%$ DMSO group using the criteria of $|\log 2(\mathrm{FC})| \geq 1.0$ (Fig. $2 \mathrm{~A}$ ). Among all the differentially expressed genes, we found that eleven genes ANGPTL4, ANKRD12, CYB5A, DCN, ECHDC3, HOXC10, MAGEB2, MT1E, TGM2, TICAM2, ZNF579, and three genes AKT1S1, MAPK14, TEN1 were down-regulated or up-regulated in a NNK dose-dependent way with a cutoff criteria of $\left.\| \log 2(\mathrm{FC})_{(0.1 \mu \mathrm{M} N N K \text { vs. } 0.1 \% \text { DMSO}}\right)^{-} \log 2(\mathrm{FC})_{(0.01 \mu \mathrm{M} N N K \text { vs. } 0.1 \% \text { DMSO })} l \geq 0.1$ (Fig. $\left.2 \mathrm{~B}\right)$.

\section{Expression of fourteen cigarette smoke exposure-related genes in KIRC and normal kidney tissues in TCGA-KIRC dataset.}

To explore the roles of these fourteen genes in KIRC, we evaluated their expression patterns in TCGA-KIRC cohort. As shown in Fig. 3, we found that three cigarette smoke exposure-reduced genes DCN, ECHDC3, MT1E and two cigarette smoke exposure-induced genes AKT1S1, TEN1 were significantly down-regulated and up-regulated, in KIRC when compared with the kidney normal tissues, respectively. However, four cigarette smoke exposure-reduced genes ANGPTL4, TGM2, TICAM2, ZNF579, and one cigarette smoke exposure-promoted gene MAPK14 were significantly up-regulated and down-regulated in KIRC when 
compared with the kidney normal tissues, respectively. In addition, MAGEB2 had very low expression levels in KIRC and kidney normal tissues.

\section{Prognostic value of individual cigarette smoke exposure-related gene in TCGA-KIRC cohort.}

Considering the opposite expression patterns of RNA-seq and TCGA and low gene expression of MAGEB2 in tissues, subsequently, eight cigarette smoke exposure-related genes ANKRD12, CYB5A, DCN, ECHDC3, HOXC10, MT1E, AKT1S1, TEN1 were selected to determine their prognostic value in KIRC cohort. The Kaplan-Meier curves showed that ANKRD12 (HR (95\%Cl): 0.64 (0.47-0.87)), CYB5A (HR (95\% Cl): 0.59 (0.43-0.80)), ECHDC3 (HR (95\%Cl): 0.45 (0.33-0.62)), and HOXC10 (HR (95\%Cl): $0.64(0.47-0.87))$ had significantly positive while DCN (HR (95\%Cl): 1.39 (1.03-1.88)) and AKT1S1 (HR (95\%Cl): 1.98 (1.452.71)) had significantly negative relationship with overall survival of KIRC patients (Fig. 4).

\section{Construction and prognostic value of cigarette smoke exposure-related gene signature in TCGA-KIRC cohort.}

A single gene was not sufficiently comprehensive and efficient to evaluate their contribution to KIRC progression, therefore, the cigarette smoke exposure-related gene signature was produced through integrating multiple candidate genes. Lasso regression analysis was firstly used to avoid overfitting problems in the gene signature, and five cigarette smoke exposure-related candidate genes ANKRD12, CYB5A, ECHDC3, MT1E, and AKT1S11 were retained when the optimal $\lambda$ value was achieved (Fig. 5A and B). Finally, a cigarette smoke exposure-related five-gene signature was established using the multivariate COX regression model and was digitized into a risk score based on the sum of the product of risk coefficient of each gene and the relevant mRNA expression level (Table 1).

Table 1

Genes included in prognostic gene signature

\begin{tabular}{|lll|}
\hline Gene & Coefficient & P value \\
\hline ANKRD12 & -0.12685 & 0.46205 \\
\hline CYB5A & -0.29841 & 0.00180 \\
\hline ECHDC3 & -0.10969 & 0.20545 \\
\hline MT1E & 0.04216 & 0.34586 \\
\hline AKT1S1 & 0.61537 & 0.00386 \\
\hline
\end{tabular}

We examined the correlation of the risk score with patients' clinicopathlogical characters, and found that the risk score was significantly higher in advanced TNM stage $(\mathbb{\nabla} / \mathbb{\nabla})$, invasion depth $T 3 / 4$, lymphatic node

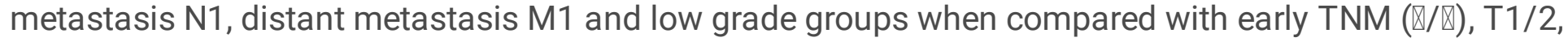
NO, MO and high grade groups (Fig. 5C). The time-dependent ROC curve was used to identify predictive value for KIRC patients' survival and revealed that the risk score had a larger area under the curve (AUC) than individual genes (Fig. 5D). 
Next, patients were classified to low-risk or high-risk group based on the median threshold of the risk score to further explore the prognostic value of the risk score in KIRC (Fig. 5E). We found that the number of deaths was significantly much more in the high-risk group than the low-risk group, and survival time of the death sample significantly decreased with the decreasing risk score (Fig. 5F). The Kaplan-Meier curve showed that the survival time of patients with high-risk score was significantly shorter than the time of patients with lower-risk score ( $\mathrm{HR}=2.12,95 \% \mathrm{Cl}$ : $1.54-2.90)$ (Fig. $5 \mathrm{G})$, and multivariate analysis showed that the risk score was an independent prognostic indicator $(\mathrm{HR}=1.7,95 \% \mathrm{Cl}: 1.25-2.30)$ after adjusting with age, sex, tumor TNM stage and grade (Fig. $5 \mathrm{H}$ ).

\section{Nomogram construction and validation.}

A prognostic nomogram can quantitatively predict an individualized prognostic risk for 1-, 3-, and 5-year overall survival by integrating cigarette smoke exposure-related gene signature risk scores with TNM stage. Each variable was assigned a corresponding point value based on its risk contribution to this model (Fig. 6A). Finally, the calibration curves suggested the agreement between the actual and predicted overall survival. The calibration curve showed that the 1-, 3-, and 5-year overall survival predicted by the nomograms were consistent with actual observations (Fig. 6B-D), indicating that the nomograms performed well.

\section{NNK exposure promoted AKT1S1 expression and activated AKT-mTOR-mediated signaling pathway.}

In this study, we found that AKT1S1 played as the most important component in the cigarette smoke exposure-related gene signature; and studies have showed that AKT1S1 acts an critical role in the intersection of the AKT-mTOR-mediated signaling pathways ${ }^{9}$, therefore, we explored AKT1S1 expression level in Ketr-3 and 786-O cells exposed to $0.1 \%$ DMSO and 0.01, 0.1 $\mu \mathrm{M}$ NNK. we found that NNK exposure dramatically up-regulated mRNA levels of AKT1S1 (Fig. 7A), and AKT1S1, p-AKT, p-mTOR protein levels had significant increase after NNK exposure when compared with the $0.1 \%$ DMSO control group (Fig. 7B).

\section{Discussion}

It's well-known that tumor cells infinite growth and metastasis are the crucial characters of tumor malignant progression ${ }^{10}$. Epidemiological data have indicated that cigarette smoking is associated with the tumor malignant progression and the poor prognosis of KIRC ${ }^{11,12}$. In this study, we found that longterm exposure to the major component of cigarette smoke, nicotine-derived NNK, increased the abilities of RCC cells colony formation and migration, which were key events of tumor growth and metastasis. Based on these findings, it was not surprising to see that cigarette smoke enhanced the malignant phenotypes of tumor cells to eventually promote KIRC progression.

There are multiple molecular events that cigarette smoke initiates and promotes the malignancy of RCC. Recently the relevance of cigarette smoke carcinogens with the inactivation of tumor suppressor genes or the activation of oncogenes has been validated for the development and progression of cancer ${ }^{13,14}$. Thus, further exploration of the molecular events involved in NNK-induced malignancy of RCC cells might 
provide new biomarkers for progression of KIRC. Here, we performed the genome-wide sequencing to seek potential biomarkers and found fourteen cigarette smoke exposure-related genes showing NNK dose-dependent down-regulation or up-regulation.

Therefore, we reasonably speculated that cigarette smoke exposure-related genes have broad prospects in progression evaluation of KIRC. Through compared the gene expression patterns in KIRC and normal kidney tissues, we found nine genes were not inconsistent with the findings in RCC cells with NNK exposure. Next, the survival analysis indicated that cigarette smoke exposure-reduced genes ANKRD12, CYB5A, ECHDC3, DCN, HOXC10 and cigarette smoke exposure-promoted gene AKT1S1 showed the positive and negative relationship with overall survival in KIRC, which was accord with some studies that decreased ANKRD12 and CYB5A and increased AKT1S1 expression show a higher frequency of tumor metastasis and are indicators of increased risk of tumor progression ${ }^{15-17}$. Though cigarette smoke exposure-reduced DCN was found to be negative prognostic factor in KIRC, increasing evidences indicate that lack of DCN expression has been regarded as an indicator of tumor metastasis ${ }^{18}$.

However, cancer heterogeneity leads to unsatisfactory effects of individual genes on the progression judgment in KIRC patients. Therefore, new efforts are urgently required to develop comprehensive estimate for KIRC. Studies have found that the gene signature will be better than a single gene to judge prognosis of a variety of tumors ${ }^{19-21}$. In this study, Lasso regression was used to screen variables to establish the prognostic model to avoid extreme prediction. The new cigarette smoke-related five-gene signature was established using the multivariate COX regression model. To provide a clinically quantitative method for gene signature, we produced a risk score based on risk coefficient of each gene and the relevant mRNA expression level. This scoring approach and its cut-off value have been confirmed to be robust in some cancer-related studies, which may be readily translated to clinical practice $22-24$.

Our data showed that the gene signature with high-risk score was significantly associated with the increased tumor invasion depth, lymphatic node metastasis, and distant metastasis and advanced TNM stage. Using the time-dependent ROC curve, we found that the risk score had a better predictive value than individual genes in KIRC prognosis. More importantly, the risk score significantly stratified patient outcomes and high-risk score was a significantly more unfavorable factor for KIRC prognosis than any single gene, indicating that the risk score had a stronger prognostic power than single genes.

Considering that AKT1S1 was the most important component of the cigarette smoke exposure-related gene signature, here we observed significantly NNK-promoted AKT1S1 expression, which was accord with some reports that elevated AKT1S1 expression in cancer cells and could contribute to tumor metastasis 17,25. AKT1S1 has been identified as an inhibitor of the mTOR complex 1 , and can be phosphorylated by AKT, and ultimately releases mTOR complex 1; AKT1S1 phosphorylation state could predict hyperactivation of the AKT-mTOR pathway in multiple cancer cell types ${ }^{9}$. In this study, we found that NNK exposure activated AKT-mTOR signaling pathway. Therefore, our data suggested that the upregulation of AKT1S1 and its related AKT-mTOR signaling pathway might play a important role in cigarette smoking-induced KIRC metastasis and progression. 
In summary, NNK exposure promoted the growth and migration abilities of RCC cells. Using RNA-seq, fourteen cigarette smoke exposure-related genes were obtained. The expression patterns showed that nine genes in KIRC when compared with normal kidney tissues were not inconsistent with the findings in RCC cells with NNK exposure, and their prognostic value were further analyzed. Five cigarette smokerelated gene signature was screened and integrated by Lasso regression analysis and multivariate COX regression model. The gene signature was more powerful than any signal gene for predicting the prognosis of KIRC patients. Moreover, NNK exposure-induced AKT1S1 and its related AKT-mTOR signaling pathway might play an important role in cigarette smoking-induced KIRC progression. Therefore, our findings provided a significant mechanistic insight into cigarette smoke-induced KIRC progression and supported that the cigarette smoke-related gene signature might serve as a highly efficient biomarker to identify metastasis and prognosis of KIRC patients.

\section{Methods}

Cell lines and reagent. Human KIRC cell line 786-O and another RCC cell line KETR-3 were purchased from the Shanghai Institute of Biochemistry and Cell Biology, Chinese Academy of Sciences (Shanghai, China). 786-O and KETR-3 cells were separately cultured in RPMI-1640 and DMEM medium supplemented with $10 \%$ fetal bovine serum (FBS), $100 \mathrm{U} / \mathrm{ml}$ penicillin and $100 \mu \mathrm{g} / \mathrm{ml}$ streptomycin. Cells were grown at $37^{\circ} \mathrm{C}$ in the presence of $5 \% \mathrm{CO}_{2}$ in a humidified incubator. NNK was purchased from Sigma-Aldrich (St Louis, $\mathrm{MO})$.

Soft agar colony formation assay. The 6-well plates were firstly coated with $0.60 \%$ agarose. Then 500 cells per well were plated in triplicate in $1 \mathrm{ml}$ of $0.35 \%$ agarose over $0.60 \%$ agarose. Cultures were fed every 3 days. At 14 days, the $0.5 \%$ NBT was used to dye the colonies. Colonies which were dyed strongly brown were scored as "positive" and colony-forming number was counted.

Wound healing assay. Cells were grown to $80 \%$ confluence into a 6 -well plate in complete medium overnight and converted to serum-free medium for another $12 \mathrm{~h}$ at $37^{\circ} \mathrm{C}$ and $5 \% \mathrm{CO}_{2}$. An injury line was made using a 2-mm-wide plastic pipette tip. Then the wells were rinsed with phosphate-buffered saline and covered with serum-free medium, and the photographs were acquired at $0 \mathrm{~h}$ and $24 \mathrm{~h}$, respectively.

Transwell assay. The transwell filter inserts with a pore size of $8 \mu \mathrm{m}$ were used for the cell migration assay. $2 \times 10^{4}$ cells (for $786-0$ ) or $5 \times 10^{4}$ cells (for KETR-3) in serum-free medium were added in the upper chamber, and placed in 24 -well plate containing $500 \mu \mathrm{l}$ complete medium. After $12 \mathrm{~h}$ incubation at $37^{\circ} \mathrm{C}$, cells in the upper chamber were carefully removed with a cotton swab and the cells that had traversed the membrane were fixed in methanol, stained with crystal violet $(0.04 \%$ in water; $100 \mu l)$, and counted the permeating cells under the inverted microscope and photographed.

Transcriptome resequencing and quantitative analysis. Human transcriptome resequencing (Vazyme Biotech) was used to analyze gene expressions collected from 786-0 cells which were exposed 
$0.1 \% \mathrm{DMSO}, 0.01 \mu \mathrm{M}, 0.1 \mu \mathrm{M}$ NNK for 120 days. The Cufflinks (cufflinks-2.2.1) was used to perform the quantitative analysis of gene expression.

Western blot analysis. Western blot was carried out as previously reported ${ }^{26}$. The anti-AKT1S1 (1:1000; Abcam, USA), anti-AKT (1:1000; CST, USA), anti-pAKT (1:1000; CST, USA), anti-mTOR (1:1000; CST, USA), anti-p-mTOR (1:1000; CST, USA), were used for primary antibody incubation at $4^{\circ} \mathrm{C}$ overnight. The antiGAPDH (1:1000; Beyotime Biotechnology, China) and anti-tubulin were used for the protein loading control.

Real-time PCR analysis. Real-time PCR was carried out in triplicate with HiScript II one step qRT-PCR SYBR Green Kit Q221-01 (Vazyme, China) according to the instruction. The complementary DNA was amplified with the following primers: AKT1S1 forward: 5'-GCCGTTGCCTCCACGACATC-3' and AKT1S1 reverse: 5'-TCATCCTCGTCCTCCTCGTTGTC-3'; GADPH forward: 5'-GCCGGT- GCTGAGTATGTC-3' and GAPDH reverse: 5'-CTTCTGGGTGGCAGTGAT-3'. GAPDH mRNA was used as an internal control for each sample, and the $\mathrm{Ct}$ value for each sample was normalized to GAPDH mRNA.

Statistical analysis. All the statistical analyses were performed by R (version 4.0.3) statistical software. The starBase project (http://starbase.sysu.edu.cn/panCancer.php) was used to analyze the gene expression pattern in KIRC and kidney normal tissues. The univariate and multivariate COX proportional regression models were performed to estimate the crude hazard ratios (HRs), adjusted HRs and their 95\% confidence intervals (Cls). Lasso regression analysis was used to screen the prognostic genes. Operating characteristic curve (ROC) was used to predict the prognostic value of genes. The Kaplan-Meier method and log-rank test were used to test the differences in survival as a function of time between the low and high risk score groups. The nomogram was developed to predict survival probability, and the fitting degree of the nomogram was evaluated by calibrations. The ANOVA analysis or Student t-test was used to evaluate the significance of quantitative data. $P$ value $<0.05$ was deemed statistically significant, and all tests were two sided.

\section{Declarations}

\section{Data availability}

The clinical features of KIRC patients can be obtained from TCGA database (http://cancergenome.nih.gov) and the corresponding RNA-sep data can be obtained downloaded from the UCSC Xena (https://xenabrowser.net/datapages/). Z.L. and Y.C. had full access to all of the data in the study and take responsibility for the integrity of the data and the accuracy of the data analysis. The datasets generated during and/or analysed during the current study are available from the corresponding author on reasonable request.

\section{Acknowledgements}


We acknowledge the phenomenal contributions of large open access genomic and clinical databases, including The Cancer Genome Atlas and starBase project, who allowed rapid progression in cancer research.

\section{Author contributions}

Z.L. and Y.C. conceived the project and designed the experiments. Y.H., Q.W., Y.T. and Z.L. conducted the experiments and data acquisition. Y.H. wrote the original draft. Q.W., Z.L. and G.S. assisted in statistical analyses and revised the paper. Y.C. supervised the study, was responsible for project administration and acquired funding. All authors read and approved the final manuscript.

\section{Funding}

This work was supported by grants from the National Natural Science Foundation of China (No. 81502772 and 81703207), the Science and Technology Department of Jiangsu Province (No. BK20150222), the China Postdoctoral Science Foundation funded project (No. 2017M611915), the Jiangsu Planned Projects for Postdoctoral Research Funds (No. 1601046B) and the Priority Academic Program Development of Jiangsu Higher Education Institutions (PAPD).

\section{Competing interests}

The authors declare no competing interests.

\section{References}

1. Siegel, R. L., Miller, K. D. \& Jemal, A. Cancer statistics, 2018. CA Cancer J Clin, 68, 7-30 (2018).

2. Chow, W. H., Dong, L. M. \& Devesa, S. S. Epidemiology and risk factors for kidney cancer. Nat Rev Urol, 7, 245-257 (2010).

3. Patel, N. H. et al. Comparative Analysis of Smoking as a Risk Factor among Renal Cell Carcinoma Histological Subtypes. J Urol, 194, 640-646 (2015).

4. Kabaria, R., Klaassen, Z. \& Terris, M. K. Renal cell carcinoma: links and risks. Int J Nephrol Renovasc Dis, 9, 45-52 (2016).

5. Hunt, J. D., van der Hel, O. L., McMillan, G. P., Boffetta, P. \& Brennan, P. Renal cell carcinoma in relation to cigarette smoking: meta-analysis of 24 studies. Int J Cancer, 114, 101-108 (2005).

6. van de Pol, J. A. A., George, L., van den Brandt, P. A., Baldewijns, M. \& Schouten, L. J. Etiologic heterogeneity of clear-cell and papillary renal cell carcinoma in the Netherlands Cohort Study.Int $J$ Cancer(2020).

7. Ehdaie, B., Furberg, H., Zabor, E. C., Hakimi, A. A. \& Russo, P. Comprehensive assessment of the impact of cigarette smoking on survival of clear cell kidney cancer. J Urol, 191, 597-602 (2014).

8. Parker, A. et al. Evaluation of the association of current cigarette smoking and outcome for patients with clear cell renal cell carcinoma. Int J Urol, 15, 304-308 (2008). 
9. Chong, Z. Z. Targeting PRAS40 for multiple diseases. Drug Discov Today, 21, 1222-1231 (2016).

10. Gonzalez Diaz, E. C., Sinha, S., Avedian, R. S. \& Yang, F. Tissue-engineered 3D models for elucidating primary and metastatic bone cancer progression. Acta Biomater, 99, 18-32 (2019).

11. Sweeney, C. \& Farrow, D. C. Differential survival related to smoking among patients with renal cell carcinoma. Epidemiology, 11, 344-346 (2000).

12. Kroeger, N. et al. Smoking negatively impacts renal cell carcinoma overall and cancer-specific survival., 118, 1795-1802 (2012).

13. Li, W. et al. Cigarette smoke enhances initiation and progression of lung cancer by mutating Notch1/2 and dysregulating downstream signaling molecules. Oncotarget, 8, 115128-115139 (2017).

14. Babu, N. et al. miRNA and Proteomic Dysregulation in Non-Small Cell Lung Cancer in Response to Cigarette Smoke. Microrna, 7, 38-53 (2018).

15. Bai, R. et al. Clinical significance of Ankyrin repeat domain 12 expression in colorectal cancer. J Exp Clin Cancer Res, 32, 35 (2013).

16. Giovannetti, E. et al. Role of CYB5A in pancreatic cancer prognosis and autophagy modulation. $J$ Natl Cancer Inst, 106, djt346 (2014).

17. Wang, C., Chen, Y., Chen, K., Zhang, L. \& Long Noncoding RNA LINC01134 Promotes Hepatocellular Carcinoma Metastasis via Activating AKT1S1 and NF-kappaB Signaling. Front Cell Dev Biol, 8, 429 (2020).

18. Sainio, A. O. \& Jarvelainen, H. T. Decorin-mediated oncosuppression - a potential future adjuvant therapy for human epithelial cancers. Br J Pharmacol, 176, 5-15 (2019).

19. Yao, Y. et al. Prognostic value of novel immune-related genomic biomarkers identified in head and neck squamous cell carcinoma.J Immunother Cancer8 (2020).

20. Gu, L., Liu, Y., Jiang, C., Sun, L. \& Zhou, H. Identification and clinical validation of metastasisassociated biomarkers based on large-scale samples in colon-adenocarcinoma. Pharmacol Res, 160, 105087 (2020).

21. Liu, H. J. et al. Ferroptosis-Related Gene Signature Predicts Glioma Cell Death and Glioma Patient Progression. Front Cell Dev Biol, 8, 538 (2020).

22. Wang, C. et al. Prognostic model and nomogram construction based on autophagy signatures in lower grade glioma.J Cell Physio/(2020).

23. Wallbillich, J. J. et al. Identification of a transcriptomic signature with excellent survival prediction for squamous cell carcinoma of the cervix. Am J Cancer Res, 10, 1534-1547 (2020).

24. Shi, R. et al. Establishment and Validation of an Individualized Cell Cycle Process-Related Gene Signature to Predict Cancer-Specific Survival in Patients with Bladder Cancer.Cancers (Basel)12 (2020).

25. Chen, K., Xu, H. \& Zhao, J. Bloom Syndrome Protein Activates AKT and PRAS40 in Prostate Cancer Cells. Oxid Med Cell Longev 2019, 3685817 (2019). 
26. Chen, Y. et al. JWA suppresses tumor angiogenesis via Sp1-activated matrix metalloproteinase-2 and its prognostic significance in human gastric cancer., 35, 442-451 (2014).

\section{Figures}
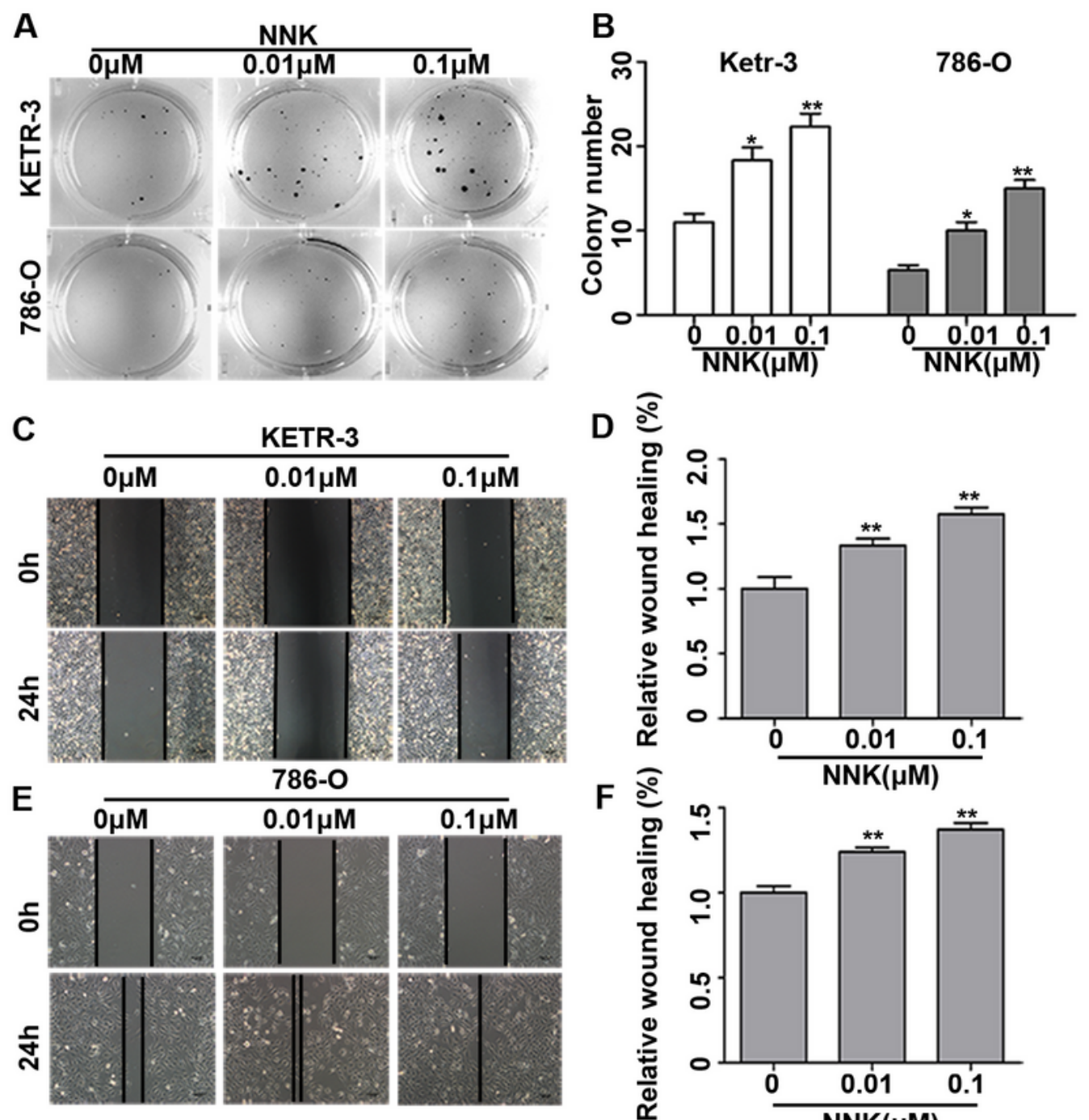

D ำ
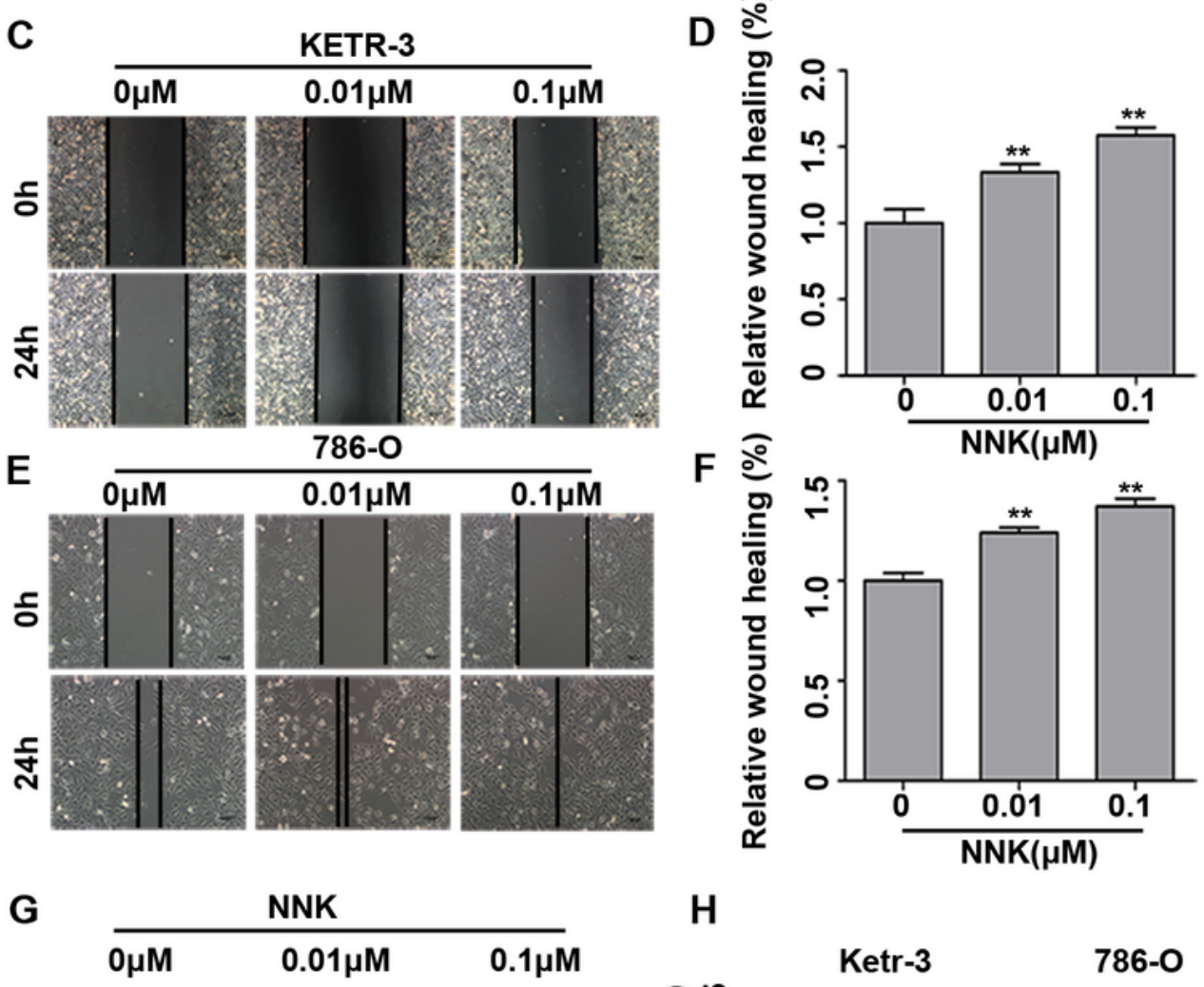

H

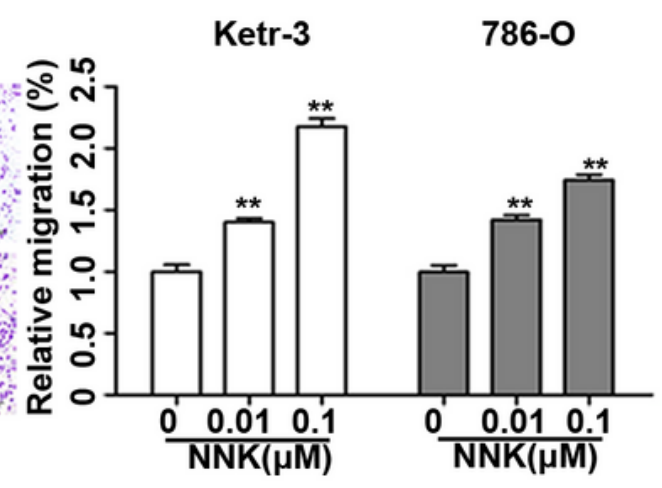

Figure 1 
NNK Exposure Increased Growth and Migration Abilities of RCC Cells. (A and B) The soft agar colony formation of KETR-3 and 786-0 cells exposed to $0(0.1 \%$ DMSO), $0.01,0.1 \mu \mathrm{M}$ NNK at passage 40 , and the number of cell colonies was counted ( $n=3$ /group). (C-F) Cell wound healing assays in KETR-3 and 786-0 cells exposed to $0(0.1 \%$ DMSO) $, 0.01,0.1 \mu \mathrm{M}$ NNK at passage 40 . The width of cell wound healing was measured ( $n=3 /$ group). Data were presented as means \pm standard deviations. ( $G$ and $H$ ) The migration of KETR-3 and 786-0 cells exposed to $0(0.1 \%$ DMSO), $0.01,0.1 \mu \mathrm{M}$ NNK at passage 40 , and the number of cell migration per field was counted in five random fields ( $n=3 /$ group). Scale bar represents $200 \mu \mathrm{m}$. Data were presented as means \pm standard deviations $* \mathrm{P}<0.05, * * \mathrm{P}<0.001$.

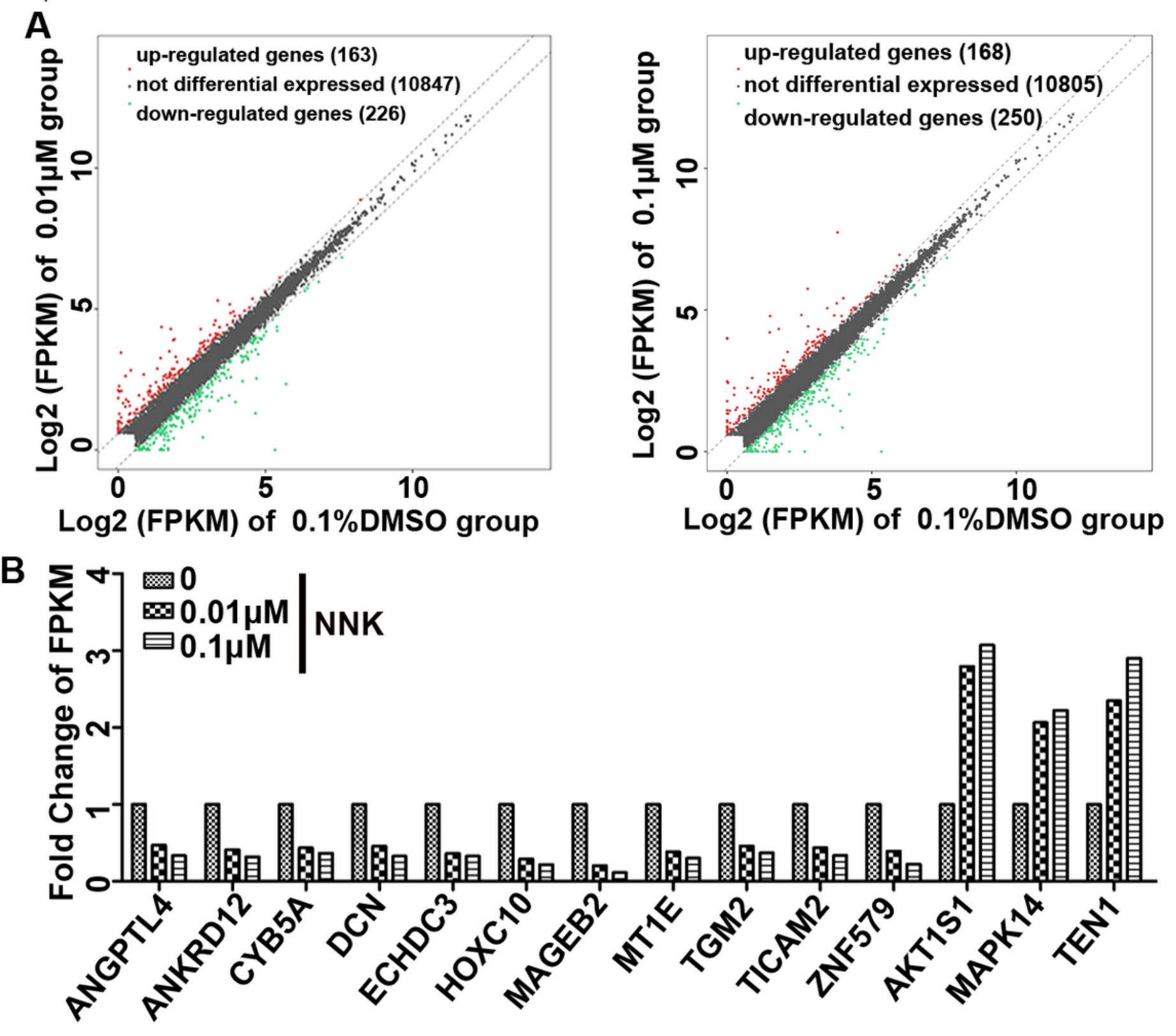

Figure 2

Identification of Cigarette Smoke Exposure-related Genes in RCC Cell. (A) The volcano plots of

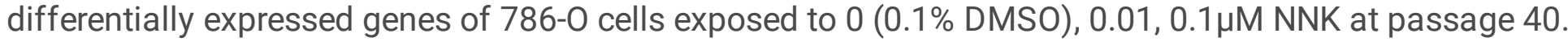


(B) Identification of Fourteen hub differentially expressed cigarette smoke exposure-related genes.
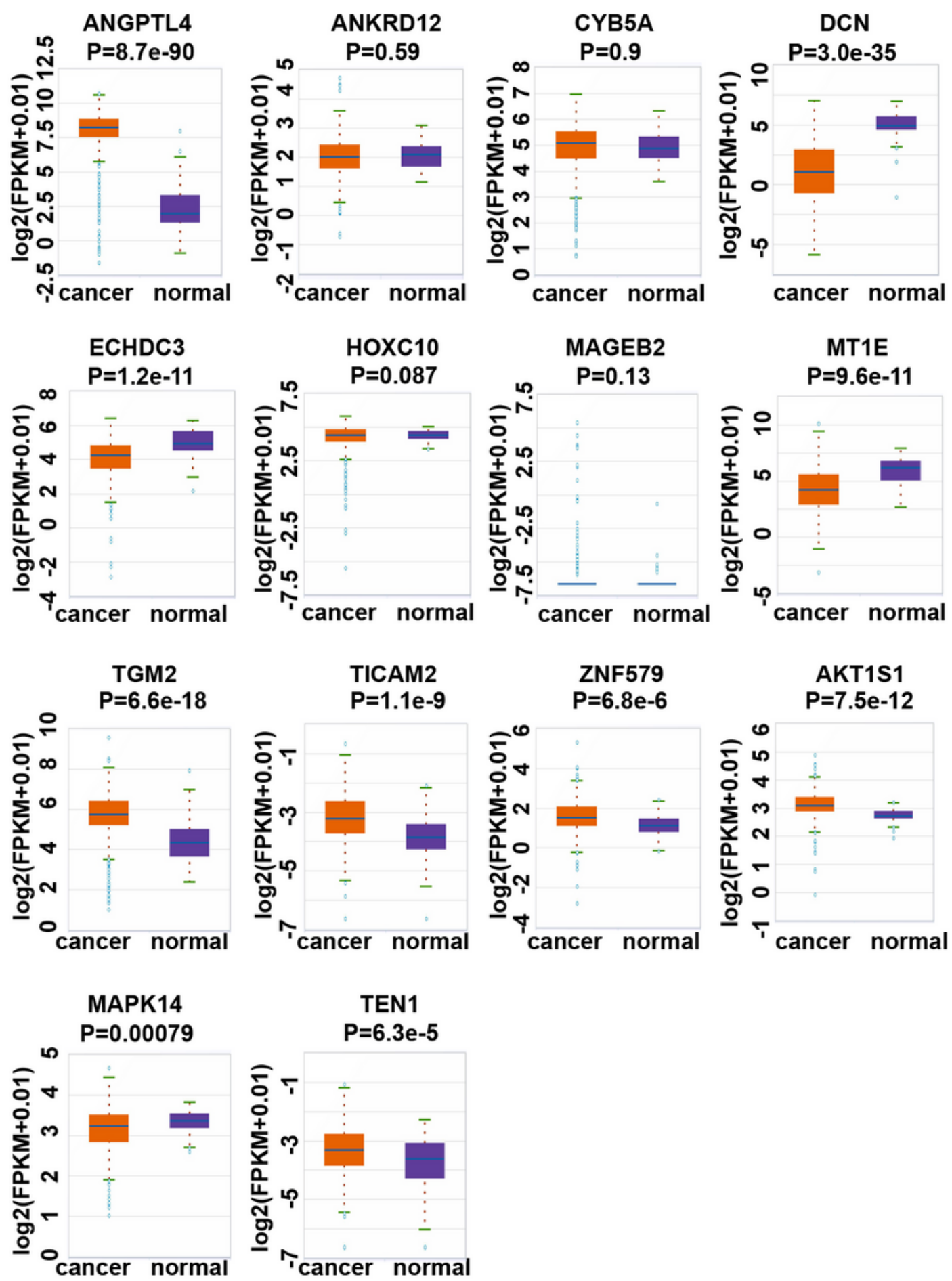

Figure 3

Expression of Fourteen Cigarette Smoke Exposure-related Genes in KIRC and normal kidney tissues in TCGA-KIRC dataset. The box plots of ANGPTL4, ANKRD12, CYB5A, DCN, ECHDC3, HOXC10, MAGEB2, MT1E, TGM2, TICAM2, ZNF579, AKT1S1, MAPK14, and TEN1 in KIRC tissues and kidney normal tissues from starBase database. 

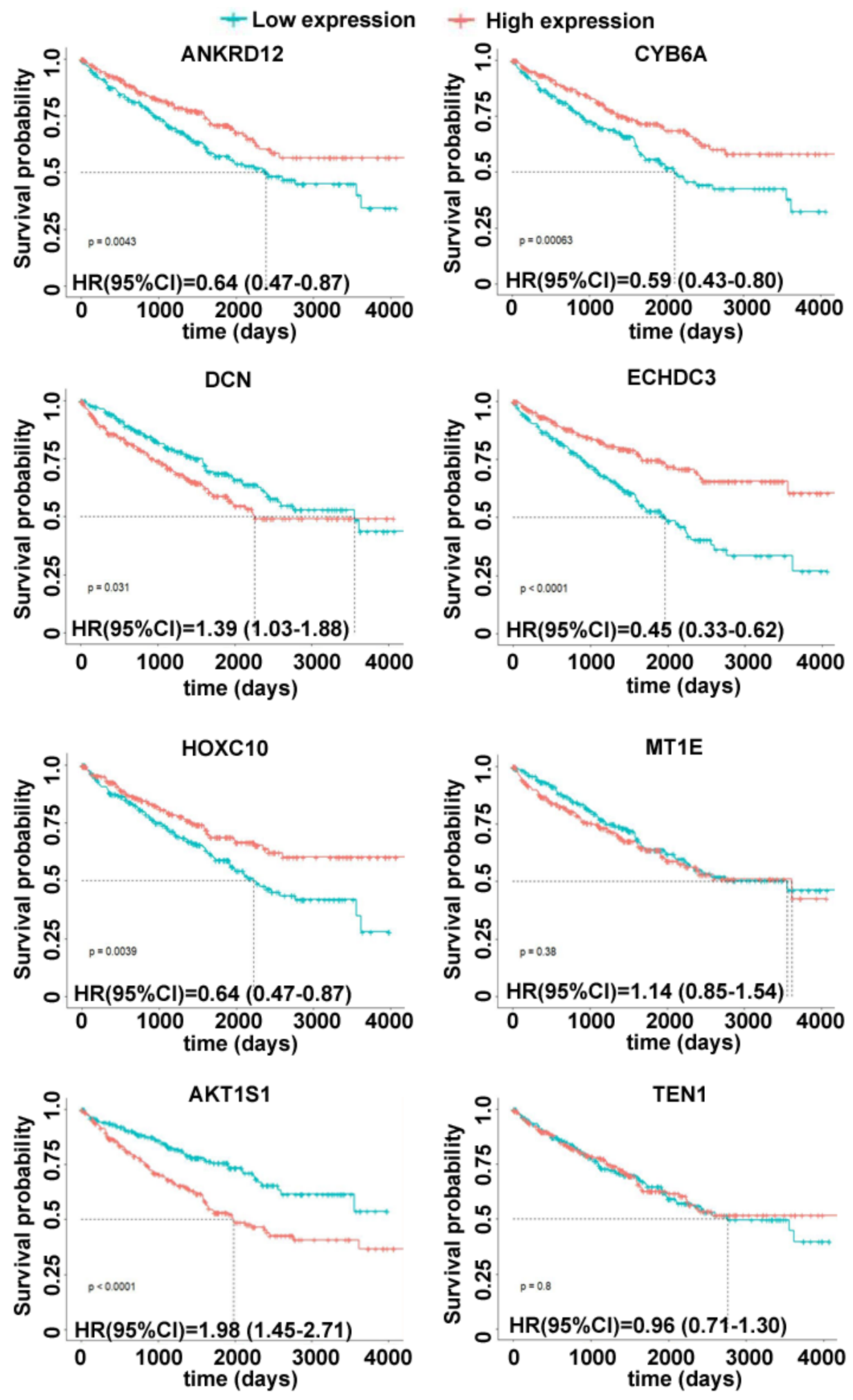

Figure 4

Prognostic Value of Individual Cigarette Smoke Exposure-related Gene In TCGA-KIRC Cohort. The KaplanMeier curves of ANKRD12, CYB5A, DCN, ECHDC3, HOXC10, MT1E, AKT1S1, TEN1 based on the median threshold of gene expression in KIRC cohort. 

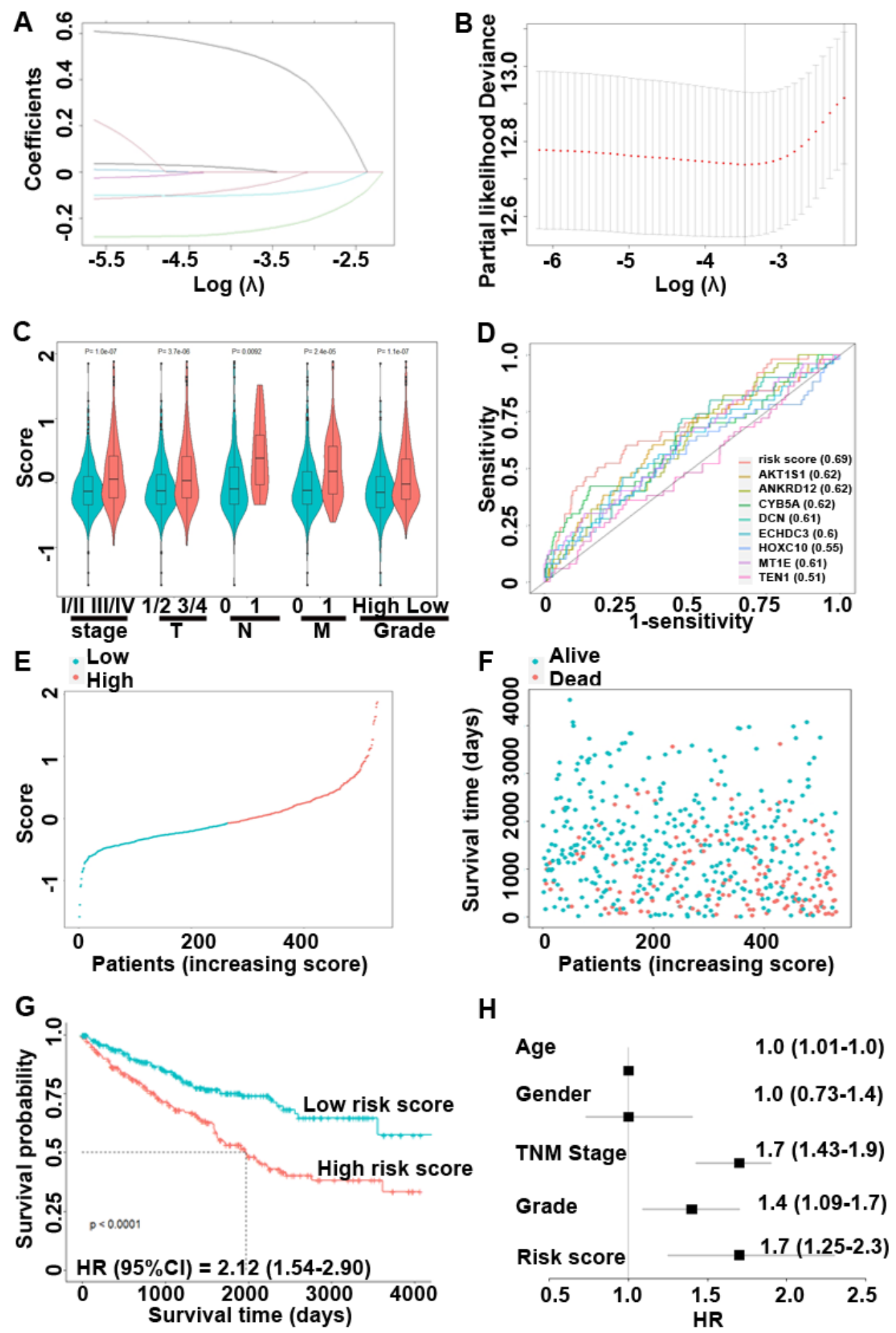

\section{Figure 5}

Construction and Prognostic Value of Cigarette Smoke Exposure-related Gene Signature in TCGA-KIRC Cohort. (A) Plots for Lasso expression coefficients of eight cigarette smoke exposure-related genes; (B) Cross-validation plot for the penalty term; (C) Violin plots of risk score in the groups of TNM stage ( $\mathbb{Q} / \mathbb{\nabla}$ vs. 『/区); invasion depth (T1/2 vs. T3/4), lymph node metastasis (N0 vs. N1), and distant metastasis (M0 vs. M1); (D) Time-dependent ROC curves of individual genes and gene signature; (E) Distribution of low-risk 
or high-risk group based on the median risk score; (F) Survival status together with overall survival of lowrisk and high-risk group; (G) Kaplan-Meier curves of the gene signature based on the median risk score; $(\mathrm{H})$ Forest plot of hazard ratios of clinicopathological features and gene signature (risk score) for overall survival of KIRC in multivariate COX regression model. ${ }^{*} \mathrm{P}<0.05$, ${ }^{*} \mathrm{P}<0.001$.

A

Points

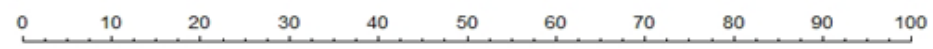

Age

Gender

TNM stage

Grade

Risk score

Total points

Linear points

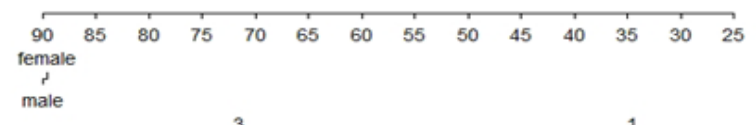

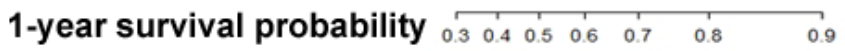

3-year survival probability

$\begin{array}{lllllllll}0.1 & 0.2 & 0.3 & 0.4 & 0.5 & 0.6 & 0.7 & 0.8 & \\ 0.9\end{array}$

5-year survival probability
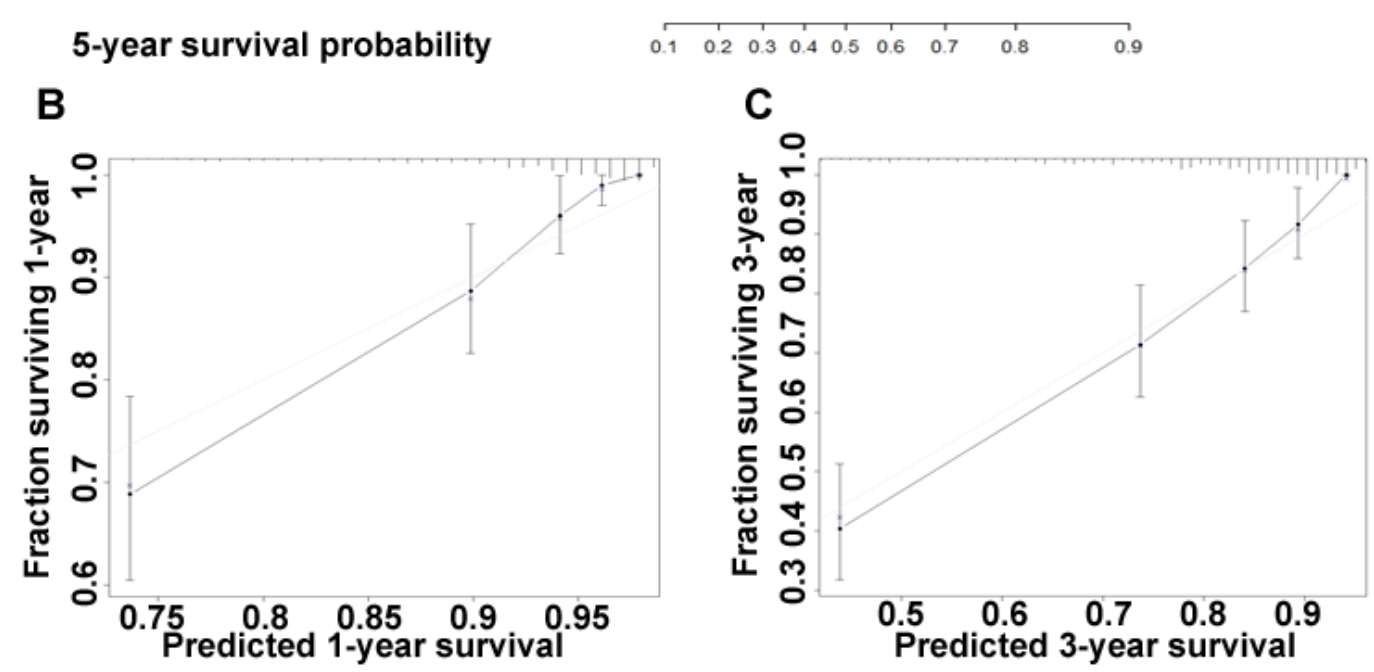

D

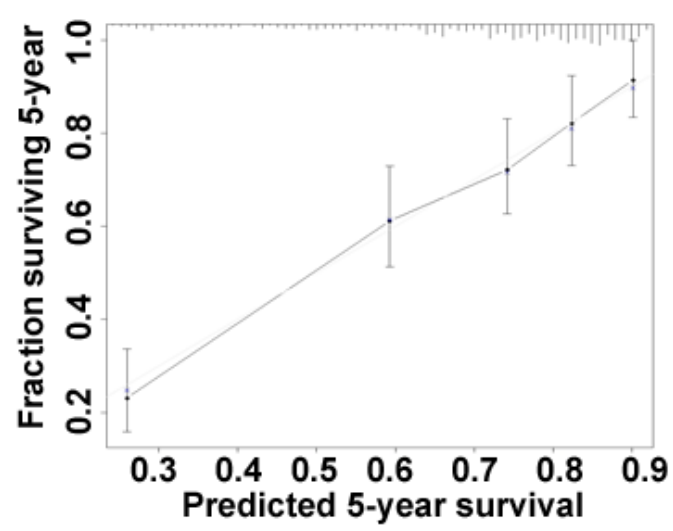

Figure 6 
Nomogram Construction and Validation. (A) The nomogram for quantitatively predicting 1-, 3-, and 5-year overall survival; (B-D) Calibration curve of the 1-, 3-, and 5-year overall survival.

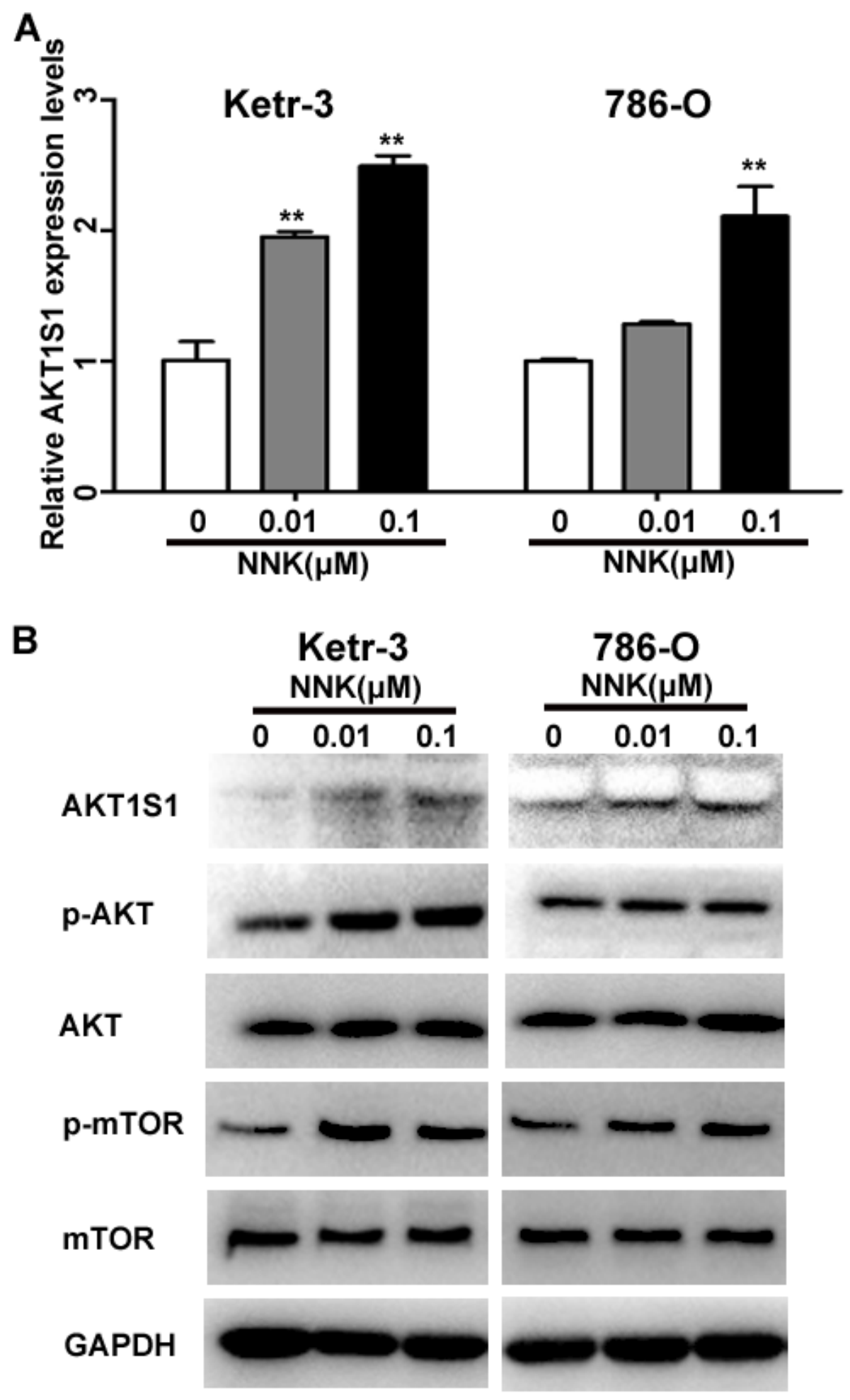

Figure 7

NNK Exposure promoted AKT1S1 expression and activated AKT-mTOR-mediated signaling pathway. (A) The mRNA expression levels of AKT1S1 in KETR-3 and 786-0 cells exposed to 0 ( $0.1 \%$ DMSO), 0.01 , 
$0.1 \mu \mathrm{M}$ NNK at passage 40; (B) The protein expression levels of AKT1S1, p-AKT, AKT, p-mTOR, GAPDH in KETR-3 and 786-O cells exposed to $0\left(0.1 \%\right.$ DMSO) $, 0.01,0.1 \mu \mathrm{M}$ NNK at passage $40 .{ }^{*} P<0.05,{ }^{\star *} P<0.001$.

\section{Supplementary Files}

This is a list of supplementary files associated with this preprint. Click to download.

- Supplementarylnformation.pdf 